
deadrise angles

M. Moore

S. Howison

J. Ockendon

J. Oliver 



\title{
Three-dimensional oblique water-entry problems at small deadrise angles
}

\author{
M. R. MOORE$E^{1} \dagger$, S. D. HOWISO N $\mathrm{N}^{1}$, J. R. OCKE $\mathrm{NDON}^{1}$ \\ AND J. M. OLIVER \\ ${ }^{1}$ Mathematical Institute, University of Oxford, 24-29 St. Giles, Oxford OX1 3LB, UK
}

(Received ?; revised ?; accepted ?. - To be entered by editorial office)

This paper extends Wagner theory for the ideal, incompressible normal impact of rigid bodies that are nearly parallel to the surface of a liquid half-space. The impactors considered are three-dimensional and have an oblique impact velocity. A variational formulation is used to reveal the relationship between the oblique and corresponding normal impact solutions. In the case of axisymmetric impactors, several geometries are considered in which singularities develop in the boundary of the effective wetted region. We present the corresponding pressure profiles and models for the splash sheets.

Key words: Authors should not enter keywords on the manuscript, as these must be chosen by the author during the online submission process and will then be added during the typesetting process (see http://journals.cambridge.org/data/relatedlink/jfmkeywords.pdf for the full list)

\section{Introduction}

The impact of a nearly-flat rigid body moving toward a liquid half-space is a canonical model for a range of physical applications, ranging from ship slamming and inkjet printing, to asteroid impact. The foundations for the theory of water-entry were first discussed by von Kármán (1929) and Wagner (1932), who were both concerned with the hydrodynamics of an alighting seaplane.

Even when neglecting physical effects such as the influence of gravity, air cushioning, surface tension, viscosity and compressibility, the normal impact of a rigid body into a fluid is a nonlinear problem and analytic progress is far from simple. However, when the impacting body is almost parallel to the undisturbed fluid free surface, that is, when the deadrise angle of the body is small, progress can be made using Wagner's idea that the bulk of the fluid motion can be approximated as that experienced due to the presence of an expanding flat plate on the undisturbed planar free surface, as explained in, for example, Armand \& Cointe (1987) and Howison et al. (1991).

The majority of analytic oblique water-entry studies consider two-dimensional impacts. In particular, there is a wealth of work concentrating on the constant speed oblique water entry of a wedge, for which there is a similarity solution. Garabedian (1953) derives the similarity solution under the assumption that the leading and trailing free surfaces separate from the wedge sides either perpendicularly or tangentially. Chekin (1989) looks at more general wedge impacts and solves the similarity problem by adapting the numerical approach of Dobrovol'skaya (1969) for normal impacts. More recently, Semenov \& Yoon

$\dagger$ This publication was based on work supported in part by Award No KUK-C1-013-04, made by King Abdullah University of Science and Technology (KAUST). 
(2009) use complex variable techniques to solve the general constant speed wedge impact problem, solving the resulting integral equations numerically. Judge et al. (2004) also look at the breakdown of oblique wedge entry and use the method of vortex distributions to approximate the impact. They compare their results to experimental observations. Both Judge et al. (2004) and Semenov \& Yoon (2009) are interested in the onset of ventilation on the trailing edge of the wedge, when the fluid detaches from the apex of the wedge above a certain tangential impact speed.

Korobkin (1988) looks at the small-time asymptotics of the oblique entry of a rigid parabola. He uses a Lagrangian description to note that to leading order, the problem reduces to the normal impact problem.

For more general impactors, Howison et al. (2004) employ the method of matched asymptotic expansions to consider two-dimensional small deadrise impacts, where the tangential component of impact velocity is on the order of the inverse of the deadrise angle. They utilise the ideas of Korobkin (1982) to transform the problem to a variational inequality, thereby revealing how the instabilities described in Howison et al. (1991) apply to the flow when the trailing boundary of the wetted region is effectively exiting the fluid.

In this paper, we concentrate on three-dimensional oblique impacts. One of the few theoretical works on three-dimensional oblique water-entry is given by Miloh (1991), where the impact of a rigid sphere is considered at small times after impact. The methodology builds on the ideas for two-dimensional oblique water-entry given in Korobkin (1988). Miloh deduces that the addition of an oblique component of impact velocity reduces the maximum downward force on the sphere, compared to the purely normal impact, suggesting that the pressure on the sphere decreases. Moreover, for moderate angles of attack (which is an indicator of the relative sizes of the oblique and normal components of the impact velocity), Miloh notes that there is very little change in the downward force on the sphere. This observation bears similarities to the analysis of Howison et al. (2004), where for horizontal impact velocities comparable to the normal impact velocity, the leading-order outer problem reduces to that for normal impact.

This paper extends the work of Moore et al. (2012), which uses the variational form of the problem to simplify the leading-order analysis. This enables us to solve the problem of impact by a general three-dimensional body and in particular, an axisymmetric body. We are able to predict the pressure on the impactor and the singular behaviour at points at which Wagner theory breaks down. We will conclude the analysis by describing the dynamics of the splash sheet (ejecta) for general axisymmetric impactors.

\section{Formulation of the problem}

We consider the impact of a rigid, smooth (except possibly at its minimum), convex body onto an initially quiescent body of fluid occupying $z^{*} \leqslant 0$ in three-dimensional Euclidean space, with axes denoted by $\left(x^{*}, y^{*}, z^{*}\right)$. The impact is assumed to begin at time $t^{*}=0$ at the origin. The fluid is assumed to be ideal and incompressible and the region not occupied by the impactor or the fluid to be a vacuum. The impactor has components of impact velocity in the positive $x^{*}-, y^{*}$ - and $z^{*}$ - directions denoted by $\dot{X}^{*}\left(t^{*}\right), \dot{Y}^{*}\left(t^{*}\right)$ and $-\dot{Z}^{*}\left(t^{*}\right)$ respectively, where a dot indicates differentiation with respect to time, so that the body profile is defined by

$$
z^{*}=L f\left(\frac{\varepsilon x^{*}-X^{*}\left(t^{*}\right)}{L}, \frac{\varepsilon y^{*}-Y^{*}\left(t^{*}\right)}{L}\right)-Z^{*}\left(t^{*}\right),
$$

where $f$ is smooth and convex, $f(0,0)=0, f$ has its minimum at $(0,0)$ and $L$ characterises the penetration depth. Throughout this paper we assume that the deadrise angle of 


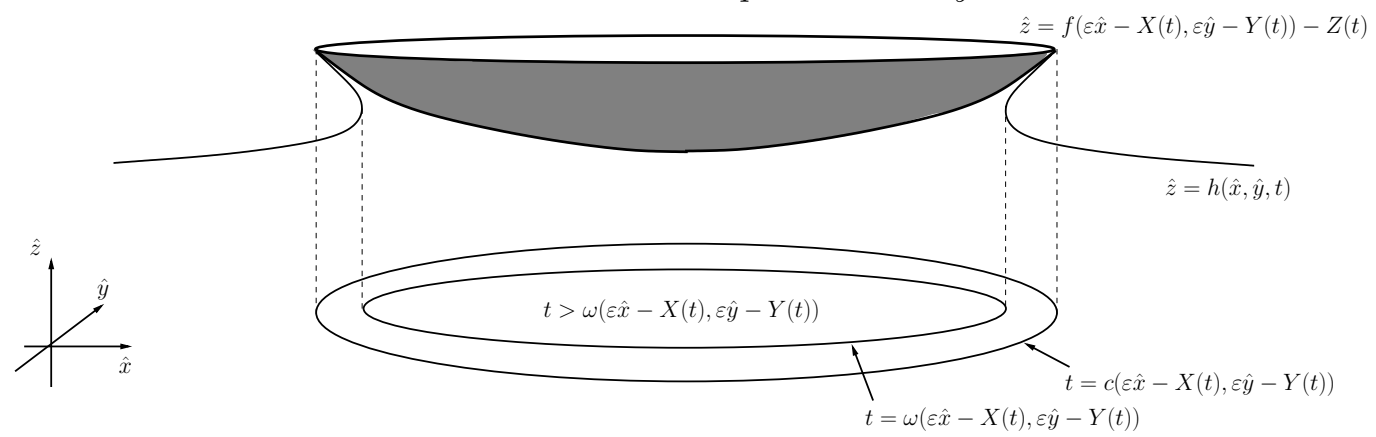

Figure 1. The splashing configuration for $t>0$. The turnover curve has projection $t=\omega(\varepsilon \hat{x}-X(t), \varepsilon \hat{y}-Y(t))$ on the $(\hat{x}, \hat{y})$-plane, with the contact set given by $t>\omega(\varepsilon \hat{x}-X(t), \varepsilon \hat{y}-Y(t))$. The curve forming the edge of the splash sheet has projection $t=c(\varepsilon \hat{x}-X(t), \varepsilon \hat{y}-Y(t))$ on the $(\hat{x}, \hat{y})$-plane.

the impactor is small, that is to say that the impactor is almost flat, so that $\varepsilon$ is a small positive constant. The prescribed functions of time $X^{*}(t), Y^{*}(t)$ and $Z^{*}(t)$ satisfy $X^{*}(0)=0, Y^{*}(0)=0, Z^{*}(0)=0$ and $\dot{Z}^{*}(0)>0$. Moreover, we assume that $\dot{X}^{*} / L$, $\dot{Y}^{*} / L$ and $\dot{Z}^{*} / L$ are all finite, at least for finite time. Note that the components of impact velocity in the $x^{*}$ - and $y^{*}$ - directions are $O(1 / \varepsilon)$ larger than the component of impact velocity in the $z^{*}$-direction. We will neglect the influence of compressibility, gravity and surface tension throughout.

We consider the dimensionless model, where distances are scaled with $L$, velocities with the initial normal impact velocity, $\dot{Z}^{*}(0)$, time with $L / \dot{Z}^{*}(0)$, velocity potential with $L \dot{Z}^{*}(0)$ and pressure with $\rho \dot{Z}^{*}(0)^{2}$, where $\rho$ is the fluid density. Using these scalings, the position of the impactor is given in dimensionless variables by

$$
\hat{z}=f(\varepsilon \hat{x}-X(t), \varepsilon \hat{y}-Y(t))-Z(t)
$$

where $X(0)=0, Y(0)=0, Z(0)=0$ and $\dot{Z}(0)>0$. The wetted extent of the impactor is defined by the curve $\partial C(t)$, which has projection $t=c(\varepsilon \hat{x}-X(t), \varepsilon \hat{y}-Y(t))$ on the $(\hat{x}, \hat{y})$ plane. The multivalued free surface is given by $\hat{z}=h(\hat{x}, \hat{y}, t)$. The turnover curve, where $\hat{z}=h$ becomes vertical, is given by $\partial \Omega(t)$, with projection $t=\omega(\varepsilon \hat{x}-X(t), \varepsilon \hat{y}-Y(t))$ on the $(\hat{x}, \hat{y})$-plane. The turnover curve is a direct three-dimensional generalisation of the turnover points as discussed in Howison et al. (1991). The set $\Omega(t)$ lying inside $\partial \Omega(t)$ has projection $t>\omega(\varepsilon \hat{x}-X(t), \varepsilon \hat{y}-Y(t))$ in the $(\hat{x}, \hat{y})$-plane. We call this the contact set. A schematic of the configuration is given in figure 1 .

The fluid is at rest initially, so that the fluid velocity is $\boldsymbol{u}=\nabla \hat{\phi}$ in terms of the potential $\hat{\phi}(\hat{x}, \hat{y}, \hat{z}, t)$, where

$$
\nabla^{2} \hat{\phi}=0
$$

in the fluid region. The kinematic condition at the body is

$$
\varepsilon f_{, 1} \frac{\partial \hat{\phi}}{\partial \hat{x}}+\varepsilon f_{, 2} \frac{\partial \hat{\phi}}{\partial \hat{y}}-\frac{\partial \hat{\phi}}{\partial \hat{z}}=\dot{Z}+\dot{X} f_{, 1}+\dot{Y} f_{, 2}
$$

on $\hat{z}=f(\varepsilon \hat{x}-X(t), \varepsilon \hat{y}-Y(t))-Z(t), t>c(\varepsilon \hat{x}-X(t), \varepsilon \hat{y}-Y(t))$, where the subscript ,$i$ denotes differentiation with respect to argument $i$. At the multivalued free surface it is given by

$$
\frac{\partial \hat{\phi}}{\partial \hat{n}}=v_{n} \quad \text { on } \quad \hat{z}=h(\hat{x}, \hat{y}, t), t<\omega(\varepsilon \hat{x}-X(t), \varepsilon \hat{y}-Y(t)),
$$


where $\partial / \partial \hat{n}$ represents the normal derivative and $v_{n}$ denotes the outward normal velocity of the free surface. The dynamic boundary condition is given by

$$
\hat{p}=-\frac{\partial \hat{\phi}}{\partial t}-\frac{1}{2}|\nabla \hat{\phi}|^{2}=0 \quad \text { on } \quad \hat{z}=h(\hat{x}, \hat{y}, t), t<\omega(\varepsilon \hat{x}-X(t), \varepsilon \hat{y}-Y(t)),
$$

in the absence of surface tension, where $\hat{p}(\hat{x}, \hat{y}, t)$ is the fluid pressure.

Finally, assuming that the free surface is planar prior to impact, the initial and far-field conditions are given by

$$
\begin{aligned}
& \hat{\phi}(\hat{x}, \hat{y}, \hat{z}, 0)=0 \quad \text { for } \quad-\infty<\hat{x}, \hat{y}<\infty, \hat{z} \leqslant 0, \\
& \hat{\phi}=O(1 / \hat{R}) \quad \text { as } \quad \hat{R}=\left(\hat{x}^{2}+\hat{y}^{2}+\hat{z}^{2}\right)^{1 / 2} \rightarrow \infty, \\
& h(\hat{x}, \hat{y}, 0)=0 \quad \text { for } \quad-\infty<\hat{x}, \hat{y}<\infty \text {, } \\
& h \rightarrow 0 \quad \text { as } \quad\left(\hat{x}^{2}+\hat{y}^{2}\right)^{1 / 2} \rightarrow \infty .
\end{aligned}
$$

Moreover, $\omega(0,0)=0$ and $c(0,0)=0$.

\subsection{Asymptotic structure}

The asymptotic structure described in Howison et al. (1991) for two-dimensional impacts at small deadrise angles extends readily to three-dimensional impacts. The problem breaks down into three distinct regions. In the outer region, of size of $O(1 / \varepsilon)$, the boundary conditions can be linearised onto the plane $z=0$, with the kinematic condition on the body applied on the expanding contact set. This is the generalisation of the flat-plate model proposed by Wagner (1932) for the constant-velocity two-dimensional impact of a wedge. Locally to $\partial \Omega(t)$, there is an inner region of size of $O(\varepsilon)$ in a plane perpendicular to $\partial \Omega(t)$ in which the free surface turns over. The flow in this region is quasi-two-dimensional provided that $\partial \Omega(t)$ is smooth. The final region is the splash sheet emanating from the inner region with extent of $O(1 / \varepsilon)$ and thickness of $O(\varepsilon)$.

Since the inner region is quasi-two-dimensional, we can state the matching conditions that must hold for the leading-order outer and splash sheet problems. For threedimensional impacts, these conditions are simply a generalisation of those given in, for example, Howison et al. (1991) or Oliver (2007).

\section{Outer region}

We neglect the splash sheet and make the outer scalings $\hat{x}=x / \varepsilon, \hat{y}=y / \varepsilon, \hat{z}=z / \varepsilon$, $\hat{\phi}=\phi / \varepsilon$ and $\hat{p}=p / \varepsilon$, so that $(2.3)$ becomes

$$
\varepsilon f_{, 1} \frac{\partial \phi}{\partial x}+\varepsilon f_{, 2} \frac{\partial \phi}{\partial y}-\frac{\partial \phi}{\partial z}=\dot{Z}+\dot{X} f_{, 1}+\dot{Y} f_{, 2} .
$$

Thus our scaling has had the desired effect that the oblique components of the velocity influence the leading-order outer problem for $\dot{X}, \dot{Y}$ of order unity; for slower horizontal velocity components, the flow would be as for normal impact, to lowest order.

When we expand $\phi, p, h$ and $\omega$ in powers of $\varepsilon$, we can linearise the boundary conditions (2.3), (2.4) and (2.5) and impose them on $z=0$ at leading-order. The resulting leadingorder outer problem is given by

$$
\begin{aligned}
\nabla^{2} \phi_{0} & =0 & & \text { in } \quad z<0, \\
\frac{\partial \phi_{0}}{\partial z} & =-\dot{Z}-\dot{X} f_{, 1}-\dot{Y} f_{, 2} & & \text { on } \quad z=0, t>\omega_{0}(x-X(t), y-Y(t)),
\end{aligned}
$$




$$
\begin{array}{ccc}
\frac{\partial \phi_{0}}{\partial z}=\frac{\partial h_{0}}{\partial t} \quad \text { on } \quad z=0, t<\omega_{0}(x-X(t), y-Y(t)), \\
\phi_{0}=0 \quad \text { on } \quad z=0, t<\omega_{0}(x-X(t), y-Y(t)),
\end{array}
$$

subject to the initial and far-field conditions (2.7)-(2.9). A subscript 0 denotes a leadingorder variable. Note that (3.5) is derived by integrating the leading-order Bernoulli equation and applying (2.6). In the outer region, $h$ refers to the lower free surface (below the turnover curve) from $\S 2$ and is hence not multivalued.

As described by Howison et al. (1991), we must also demand that the velocity potential has square-root behaviour in distance from the turnover curve as we approach it in any perpendicular plane. Our final requirement is that the Wagner condition must hold at the turnover curve:

$$
h_{0}(x, y, t)=f(x-X(t), y-Y(t))-Z(t) \quad \text { on } \quad t=\omega_{0}(x-X(t), y-Y(t)) .
$$

This states that the leading-order outer free surface meets the impactor at the leadingorder turnover curve.

\subsection{Displacement potential formulation}

It is convenient to transform the problem (2.6)-(2.9), (3.2)-(3.6) using the leading-order displacement potential, as first introduced in the context of impact problems by Korobkin (1982), which is defined by

$$
\Psi(x, y, z, t)=-\int_{0}^{t} \phi_{0}(x, y, z, \tau) \mathrm{d} \tau .
$$

By the definition of the turnover curve, the contact set is given by $t>\omega_{0}(x-X(t), y-$ $Y(t))$ and the free surface by $t<\omega_{0}(x-X(t), y-Y(t))$. Hence, under the transformation (3.7), the kinematic boundary condition on the body, (3.3), becomes

$$
\begin{aligned}
\frac{\partial \Psi}{\partial z}(x, y, 0, t) & =-\int_{0}^{\omega_{0}} \frac{\partial h_{0}}{\partial \tau}(x, y, \tau) \mathrm{d} \tau+\int_{\omega_{0}}^{t} \dot{Z}(\tau)+\dot{X}(\tau) f_{, 1}+\dot{Y}(\tau) f_{, 2} \mathrm{~d} \tau \\
& =-h_{0}\left(x, y, \omega_{0}(x-X(t), y-Y(t))\right)+\left[Z(t)-Z\left(\omega_{0}(x-X(t), y-Y(t))\right)\right] \\
& =Z(t)-f(x-X(t), y-Y(t))
\end{aligned}
$$

where in the first line, the range of integration is split into $\tau<\omega_{0}(x-X(\tau), y-Y(\tau))$, where we apply (3.4), and $\tau>\omega_{0}(x-X(\tau), y-Y(\tau))$, where we use (3.3). In the second line we have used (2.8) and in the final line we have applied (3.6).

Thus it is readily shown that the displacement potential problem is given by (dropping the subscripts on the leading-order variables)

$$
\begin{aligned}
\nabla^{2} \Psi & =0 & & \text { in } \quad z<0, \\
\frac{\partial \Psi}{\partial z} & =Z(t)-f(x-X(t), y-Y(t)) & & \text { on } \quad z=0, t>\omega(x-X(t), y-Y(t)), \\
\frac{\partial \Psi}{\partial z} & =-h & & \text { on } \quad z=0, t<\omega(x-X(t), y-Y(t)),(3) \\
\Psi & =0 & & \text { on } \quad z=0, t<\omega(x-X(t), y-Y(t)),(3 .
\end{aligned}
$$

subject to

$$
\Psi=O(1 / R) \quad \text { as } \quad R=\left(x^{2}+y^{2}+z^{2}\right)^{1 / 2} \rightarrow \infty
$$

and the far-field condition on $h$ given by (2.9). Finally, we require $\Psi$ to have a $(3 / 2)$-power singularity in distance from the turnover curve as we approach it in any perpendicular plane. 
The problem (2.8), (2.9), (3.8)-(3.12) contains no time derivatives. Therefore, under the transformation

$$
x-X(t) \mapsto x, y-Y(t) \mapsto y,
$$

the displacement potential formulation reduces exactly to the equivalent normal impact formulation. Hence, given a solution to the normal impact problem we are able to write down the solution to the corresponding oblique impact. In particular, if $\Psi(x, y, z, t)$, $z=h(x, y, t)$ and $t=\omega(x, y)$ are, respectively, the leading-order outer displacement potential, free surface and turnover curve for the normal impact of the body profile $z=f(x, y)-Z(t)$, then $\Psi(x-X(t), y-Y(t), z, t), z=h(x-X(t), y-Y(t), t)$ and $t=\omega(x-X(t), y-Y(t))$ are the leading-order outer displacement potential, free surface and turnover curve for the oblique impact of the body $z=f(x-X(t), y-Y(t))-Z(t)$. This is a direct analogue of the conclusion for two-dimensional oblique impact problems, given in Moore et al. (2012). We note, however, that the leading-order outer velocity potential and leading-order outer pressure do not have such a simple relation with their normal-impact counterparts, due to the time-dependency of this moving frame.

We now exploit this analysis to investigate the oblique impact of axisymmetric bodies.

\section{Axisymmetric impactors}

\subsection{Normal impact}

The normal water-entry of an axisymmetric impactor is considered in Howison et al. (1991). We solve the displacement potential formulation here by setting $X(t)=0=Y(t)$ in $(2.8),(2.9),(3.8)-(3.12)$. In cylindrical polar coordinates $(r, \theta, z)$, Laplace's equation for the displacement potential becomes

$$
\frac{1}{r} \frac{\partial}{\partial r}\left(r \frac{\partial \Psi}{\partial r}\right)+\frac{\partial^{2} \Psi}{\partial z^{2}}=0 \quad \text { in } \quad z<0 .
$$

The boundary conditions (3.9)-(3.11) reduce to

$$
\begin{aligned}
& \frac{\partial \Psi}{\partial z}=Z(t)-f(r) \quad \text { on } \quad z=0, r<d(t), \\
& \frac{\partial \Psi}{\partial z}=-h \quad \text { on } \quad z=0, r>d(t) \text {, } \\
& \Psi=0 \quad \text { on } z=0, r>d(t),
\end{aligned}
$$

where $f(r)$ is the axisymmetric body profile and $r=d(t)$ is the leading-order position of the turnover curve. These are subject to

$$
\begin{array}{lll}
\Psi=O(1 / R) & \text { as } & R=\left(r^{2}+z^{2}\right)^{1 / 2} \rightarrow \infty, \\
h \rightarrow 0 & \text { as } \quad r \rightarrow \infty,
\end{array}
$$

and $d(0)=0$. We also have the requirement that $\Psi$ has $(3 / 2)$-power behaviour as we approach the turnover curve $r=d(t), z=0$ in any plane perpendicular to it.

We can find separable solutions and hence write the general solution as a superposition of these of the form

$$
\Psi(r, z, t)=\int_{0}^{\infty} \alpha(\lambda) e^{\lambda z} J_{0}(\lambda r) \mathrm{d} \lambda,
$$

where $J_{0}(\lambda r)$ is the Bessel function of the first kind of order zero. The unknown function $\alpha(\lambda)$ can be determined by applying (4.2) and (4.4). We deduce the following dual integral 
equations:

$$
\begin{array}{ll}
\int_{0}^{\infty} \alpha(\lambda) J_{0}(\lambda r) \mathrm{d} \lambda=0 & \text { for } \quad r>d(t), \\
\int_{0}^{\infty} \lambda \alpha(\lambda) J_{0}(\lambda r) \mathrm{d} \lambda=Z(t)-f(r) & \text { for } \quad r<d(t),
\end{array}
$$

which can be solved by adapting the method of Sneddon (1966, pp. 76-77) to show that

$$
\alpha(\lambda)=\int_{0}^{d(t)} \chi(\sigma) \sin \lambda \sigma \mathrm{d} \sigma
$$

where

$$
\chi(\sigma)=\frac{2}{\pi} \int_{0}^{\sigma} \frac{r(Z(t)-f(r))}{\sqrt{\sigma^{2}-r^{2}}} \mathrm{~d} r .
$$

Then (4.7), (4.10) and (4.11) give the displacement potential solution for the normal impact of the body $z=f(x, y)-Z(t)$.

To solve for the location of the turnover curve, we must meet the requirement that the displacement potential have (3/2)-power behaviour in distance as we approach the turnover curve. Upon evaluating the displacement potential on $z=0$ and writing $r=$ $d(t)-\delta$, we find that

$$
\begin{aligned}
\Psi(d(t)-\delta, 0, t)= & \delta^{1 / 2} \int_{0}^{1} \frac{\chi(d(t))}{\sqrt{2 d(t)(1-S)}} \mathrm{d} S+ \\
& \delta^{3 / 2} \int_{0}^{1} \frac{\chi(d(t))(S+1)-4 S d(t) \chi^{\prime}(d(t))}{4 d(t) \sqrt{2 d(t)(1-S)}} \mathrm{d} S+O\left(\delta^{5 / 2}\right)
\end{aligned}
$$

as $\delta \downarrow 0$. The coefficient of the $\delta^{1 / 2}$ term must vanish, so that we require

$$
\chi(d(t))=0,
$$

where $\chi(\sigma)$ is given by (4.11). Note that it follows that

$$
\Psi(r, 0, t)=-\frac{4 \chi^{\prime}(d(t))}{3 \sqrt{2 d(t)}}(d(t)-r)^{3 / 2}+O\left((d(t)-r)^{5 / 2}\right),
$$

as $r \uparrow d(t)$.

Thus, having deduced expressions for $\Psi(r, z, t)$ and $d(t)$, we can find the leading-order outer free surface by using (4.3) to deduce that

$$
h(r, t)=-\int_{0}^{d(t)} \frac{\chi^{\prime}(\sigma)}{\sqrt{r^{2}-\sigma^{2}}} \mathrm{~d} \sigma .
$$

\subsection{Oblique impact}

With these expressions for $\Psi(r, z, t), h(r, t)$ and $d(t)$ in the case of the normal impact of the body $z=f(r)-Z(t)$, we can use the argument at the end of $\S 3.1$ to deduce the form of the leading-order displacement potential, leading-order outer free surface and leading-order turnover curve in the impact of the body $z=f(x-X(t), y-Y(t))-Z(t)$ by making the change of variables given in (3.13) in reverse. Hence, we simply have to write

$$
r=\sqrt{(x-X(t))^{2}+(y-Y(t))^{2}}
$$

in the expressions (4.7), (4.10), (4.11), (4.13) and (4.15).

Clearly, the turnover curve is simply a circle moving in the direction $(X(t), Y(t))$ in the 
$(x, y)$-plane. However, we must remember that, as in the case of two-dimensional oblique impacts, this analysis is only valid if the turnover curve is everywhere advancing. This is a consequence of the local-in-space-and-time linear stability analysis of Howison et al. (1991), who argue that not only does the two-dimensional problem become unstable to out-of-plane perturbations in the vicinity of the turnover curve when the turnover curve is retreating, but also that solving the hyperbolic equation for the leading-order outer free surface is no longer possible, as we lose causality when we try to find the boundary of the contact set. Therefore, for our analysis to be valid, we require the outward normal speed of the turnover curve to be positive. In particular, if the turnover curve is defined by $F(x, y, t)=0$, then its outward normal speed is given by

$$
v_{n}=-\frac{1}{|\nabla F|} \frac{\partial F}{\partial t}
$$

so that with $F(x, y, t)=\sqrt{(x-X(t))^{2}+(y-Y(t))^{2}}-d(t)$, we have

$$
v_{n}(\theta, t)=\dot{d}(t)+\dot{X}(t) \cos \theta+\dot{Y}(t) \sin \theta,
$$

where $\theta$ represents polar angle around the turnover curve. Hence, it is possible that there are values of $\dot{X}, \dot{Y}, \theta$ and $t$ such that $v_{n} \leqslant 0$. To get a clearer picture of this, we consider two different examples of oblique impact. For the sake of simplicity, we assume that $X(t)=U t, Y(t)=0$ and $Z(t)=t$, where $U>0$, in both of the examples, although our results generalise readily.

\subsection{Oblique impact of a cone}

We consider the oblique impact of a cone defined by $z=\beta r-t$ where $\beta>0$. For the moment we ignore the fact that, as in the two-dimensional oblique entry of a wedge, there is an infinite negative pressure at the apex of the cone, but we discuss cavitation in $\S 5$.

Upon integrating (4.11), we find that

$$
\chi(\sigma)=\frac{2}{\pi}\left(\sigma t-\frac{\beta \sigma^{2} \pi}{4}\right),
$$

so that upon applying the consistency condition, (4.13), we find

$$
r=\sqrt{(x-U t)^{2}+y^{2}}=\frac{4 t}{\beta \pi}=d(t),
$$

defines the location of the turnover curve. Note that, when $U=0$, this reduces to the normal impact case as deduced from the velocity potential solution in Shiffman \& Spencer (1951) and Howison et al. (1991). Using (4.17), we deduce that the outward normal speed of the turnover curve is given by

$$
v_{n}=\frac{4}{\beta \pi}+U \cos \theta .
$$

The normal impact speed is independent of $t$, reflecting the fact that, in this example, the problem (2.8), (2.9), (3.8)-(3.12) admits a similarity solution. More interestingly, the turnover curve is only advancing for $U<\min _{\theta \in(\pi / 2,3 \pi / 2)}-4 /(\beta \pi \cos \theta)$. Thus, we expect the turnover curve to stop advancing at its trailing edge, $\theta=\pi$, when $U=4 /(\beta \pi)$.

Hence, the solution is valid for all time for $0<U<4 /(\beta \pi)$, but breaks down at a critical horizontal velocity. 


\subsection{Oblique impact of a blunt power-law body}

We consider a blunt power-law body with profile of the form $z=\beta r^{n}-t$, where $\beta>0$ and $n>1$. Integrating (4.11) for $\chi(\sigma)$, we deduce that

$$
\chi(\sigma)=\frac{2}{\pi}\left(\sigma t-\beta 2^{n} \sigma^{n+1} B\left(\frac{n+2}{2}, \frac{n+2}{2}\right)\right),
$$

where $B(\cdot, \cdot)$ is the beta function. Therefore, the turnover curve is given by

$$
r=\sqrt{(x-U t)^{2}+y^{2}}=\left(\frac{t}{\beta 2^{n} B((n+2) / 2,(n+2) / 2)}\right)^{1 / n}=d(t) .
$$

Its outward normal speed is

$$
v_{n}=\frac{1}{n}\left(\frac{1}{2^{n} \beta B((n+2) / 2,(n+2) / 2)}\right)^{1 / n} t^{1 / n-1}+U \cos \theta,
$$

so that $v_{n}>0$ for all $U$, when $t>0$ is sufficiently small, but the normal speed of the turnover curve vanishes when

$$
t=t_{c}=\min _{\theta \in(\pi / 2,3 \pi / 2)}\left[-n U \cos \theta\left(2^{n} \beta B((n+2) / 2,(n+2) / 2)\right)^{1 / n}\right]^{n /(1-n)} .
$$

Again, this first occurs on $\theta=\pi$, and hence the turnover curve stops advancing on the ray $\theta=\pi$ at time

$$
t_{c}=\left[n U\left(2^{n} \beta B((n+2) / 2,(n+2) / 2)\right)^{1 / n}\right]^{n /(1-n)} .
$$

For large values of $n$ with $U, \beta$ fixed, the asymptotic form of the critical time is

$$
t_{c} \sim \frac{1}{n}\left(\frac{2}{\pi}\right)^{1 / 2 n}(n+2)^{1 / 2 n}
$$

so that the flatter the body profile, the more rapid the breakdown. A plot of the critical time as a function of the exponent, $n$, is shown in figure 2 .

\section{Breakdown and cavitation in the outer region}

Given the expansion of the displacement potential as we approach the turnover curve in (4.14), we can work out the coefficient of the square-root singularity in the leadingorder outer velocity potential and the corresponding coefficient of the inverse square-root singularity in leading-order outer pressure on the body. By noting that $\phi=-\Psi_{t}$, we have that $\phi(r, 0, t)=S(\theta, t)(d(t)-r)^{1 / 2}+O\left((d(t)-r)^{3 / 2}\right)$ as $r \uparrow d(t)$, where

$$
S(\theta, t)=\sqrt{\frac{2}{d(t)}} \chi^{\prime}(d(t)) v_{n}(\theta, t) .
$$

Therefore, we can express the leading-order outer pressure on the impactor in the vicinity of the turnover curve in terms of $S(\theta, t)$ and $v_{n}(\theta, t)$ by

$$
p(r, 0, t)=-\frac{1}{2} S(\theta, t) v_{n}(\theta, t)(d(t)-r)^{-1 / 2}+O\left((d(t)-r)^{1 / 2}\right),
$$

as $r \uparrow d(t)$. Hence, when $v_{n}=0$, the coefficients of the inverse square-root singularities in the leading-order outer velocity and pressure vanish. This is also the case in the breakdown of two-dimensional impacts, as discussed by Moore et al. (2012).

Explicit forms of (5.2) are: 


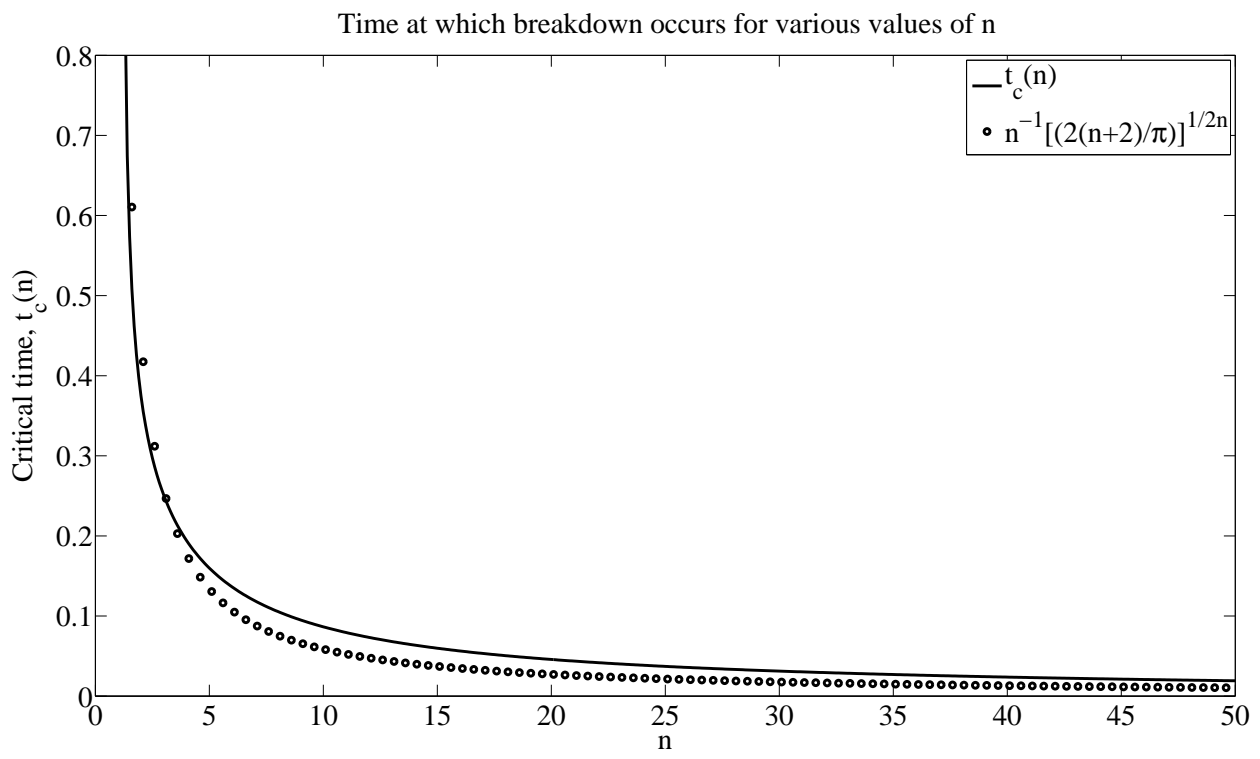

Figure 2. The time of breakdown $t_{c}$ as a function of the exponent of the impactor profile, $n$. The dotted line indicates the asymptotic form of the critical time, as given in (4.25).

- for a cone $z=\beta r$

$p(r, 0, t)=-\frac{U^{2} \beta}{2} \cosh ^{-1}\left(\frac{4 t}{\beta \pi r}\right)+\frac{2 t}{\pi \sqrt{(4 t / \beta \pi)^{2}-r^{2}}}\left(\left(\frac{4}{\beta \pi}\right)^{2}+\frac{2 U}{t} r \cos \theta+U^{2} \cos ^{2} \theta\right)$,

for $0<r=\sqrt{(x-U t)^{2}+y^{2}}<d(t)=4 t /(\pi \beta)$. We plot the cone pressure profile in figure 3 for various values of $U$, where we have chosen $\beta=4 / \pi$ so that breakdown occurs at $U=1$. When we give the cone a forward velocity component, there is a region of negative pressure on the impactor inside the turnover curve. This forms for any $U>0$ due to the aforementioned local corner flow at the apex of the cone. As $U$ increases, the isobar of zero pressure gradually spreads further from the apex, until at breakdown, it touches the turnover curve on the ray $\theta=\pi$.

- for a paraboloid $z=\beta r^{2}$

$$
p(r, 0, t)=\frac{1}{\sqrt{3 t / 2 \beta-r^{2}}}\left(\frac{3}{2 \beta \pi}+\frac{4 r U \cos \theta}{\pi}+\frac{8 \beta U^{2} r^{2} \cos ^{2} \theta}{3 \pi}\right)-\frac{8 \beta U^{2}}{3 \pi} \sqrt{\frac{3 t}{2 \beta}-r^{2}},
$$

for $0<r=\sqrt{(x-U t)^{2}+y^{2}}<d(t)=\sqrt{3 t /(2 \beta)}$. We plot the paraboloid pressure profile in figure 4 . We consider the case where $U=1$ and the parameters are chosen so that the corresponding breakdown time is given by $t_{c}=1$. Prior to breakdown, a region of negative pressure forms on the trailing side of the paraboloid. As we approach breakdown, the zero pressure isobar touches the turnover curve; this behaviour indeed occurs for all the geometries discussed in $\S 4$.

These observations suggest that it is possible that cavitation occurs before the turnover curve stops advancing. In particular, there is the possibility of a patch cavity forming in the region of negative pressure. The introduction of such a cavity into the model would make the solution of the leading-order outer problem somewhat more complex. We do 

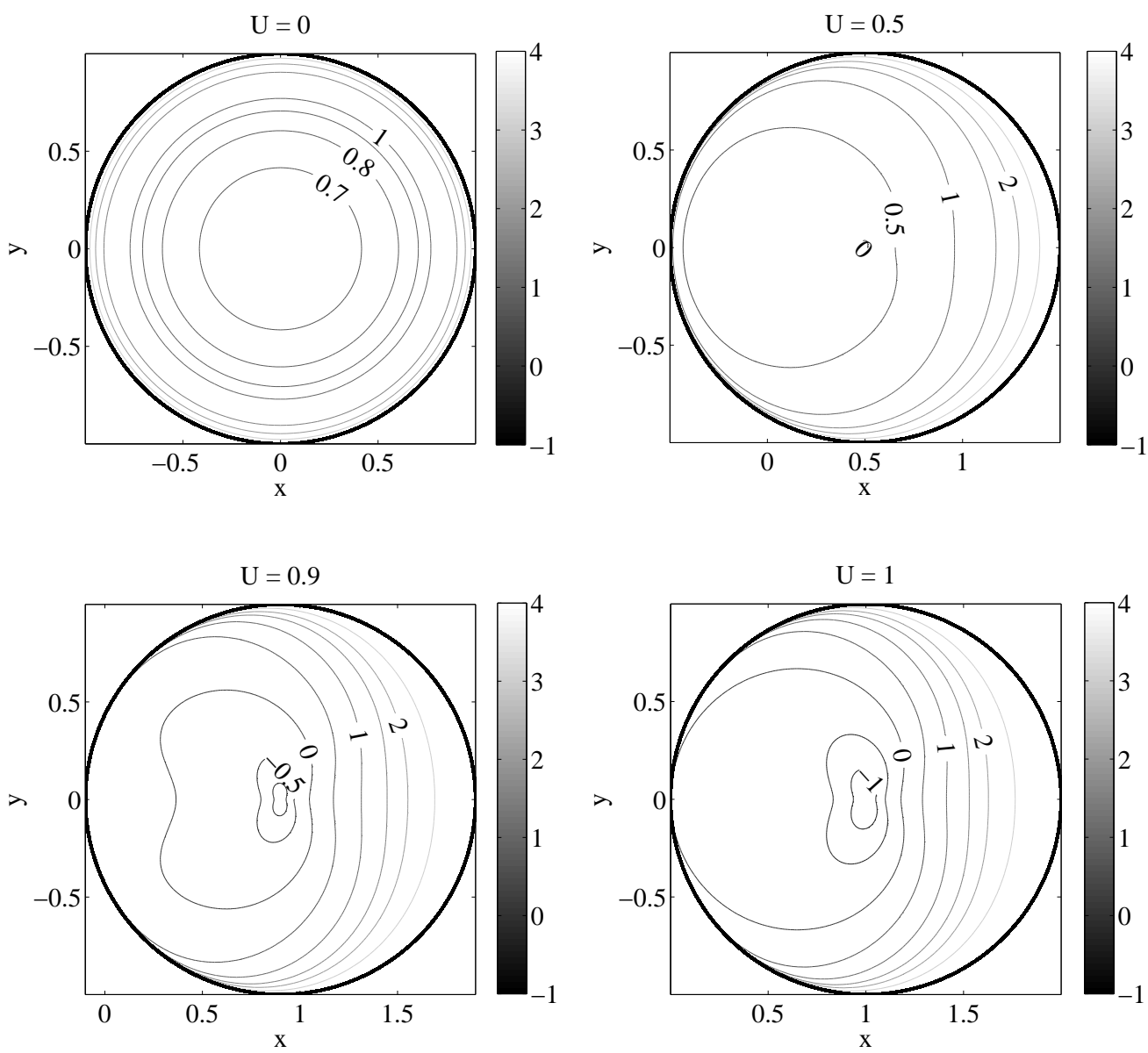

Figure 3. Isobars of the leading-order outer pressure on a cone for different values of $U$. The turnover curve is depicted in bold black. We have truncated the contour domain for ease of viewing the more interesting behaviour on the interior of the turnover curve. As we increase $U$ from zero, corresponding to the normal impact of a cone (in the top-left corner), to 1 , corresponding to the critical forward velocity (bottom-right), we see a significant change in the pressure profile. The region of negative pressure spreads on the trailing side of the cone. At breakdown, the isobar of zero pressure touches the turnover curve.

not consider ideas of cavitation any further here, but we will propose a model that could allow for cavitation in the Appendix.

\section{Splash sheet region}

The three-dimensional oblique splash sheet problem is a generalisation of that described for normal impact problems in Oliver (2002). The splash sheet is ejected from the turnover region and lies an order unity distance from the leading-order turnover curve $t=\omega_{0}(x, y), z=0$.

The splash sheet is slender, with thickness of the order of the deadrise angle and extent of the order of the inverse of the deadrise angle. Hence, if $\left(\xi_{1}, \xi_{2}\right)$ are local orthogonal 

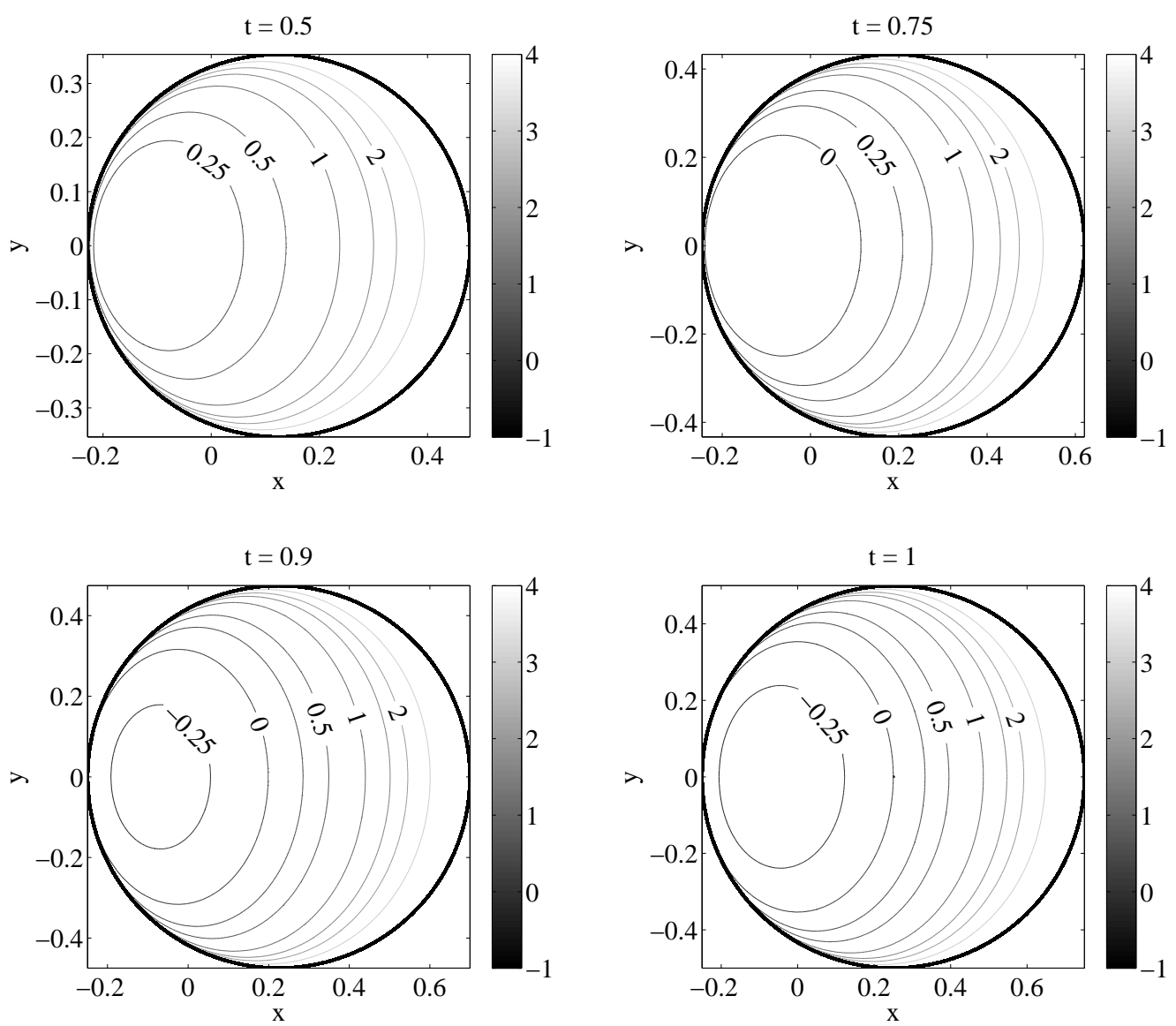

FIGURE 4. Isobars of the leading-order outer pressure on a paraboloid for different times. The turnover curve is depicted in bold black. We have again truncated the contour domain for ease of viewing the more interesting behaviour on the interior of the turnover curve. As we increase time, a region of negative pressure forms on the trailing side of the paraboloid. This region appears before breakdown occurs and grows in size as we increase time. At breakdown, the isobar of zero pressure touches the turnover curve.

curvilinear coordinates based on the impactor (scaled with $1 / \varepsilon$ ), then $(\bar{u}, \bar{v})$, which are the velocity components in the $\xi_{1}$ - and $\xi_{2}$-directions respectively (scaled with $1 / \varepsilon$ ), and the sheet thickness $\bar{h}$ (scaled with $\varepsilon$ ) must satisfy the zero-gravity shallow water equations

$$
\frac{\partial \bar{h}}{\partial t}+\nabla \cdot(\overline{\boldsymbol{u}} \bar{h})=0, \quad \frac{\partial \overline{\boldsymbol{u}}}{\partial t}+(\overline{\boldsymbol{u}} \cdot \nabla) \overline{\boldsymbol{u}}=0
$$

to leading-order $\dagger$, where the gradient operator, $\nabla$, is based on the local coordinates. Since the extent of the splash sheet is much smaller than the principal radii of curvature of the impactor, to leading-order we can take, without loss of generality, $\left(\xi_{1}, \xi_{2}\right)$ to be Cartesian with the same orientation and origin as in the outer region.

$\dagger$ It can be shown that these are the leading-order equations governing the splash sheet problem even if the frame is accelerating. 
The boundary conditions for the problem are found through matching with the inner region. As described in Oliver (2002), this implies

$$
(\bar{u}, \bar{v})=2 v_{n} \boldsymbol{n} \quad \text { at } \quad t=\omega(x-X(t), y-Y(t)),
$$

where $\boldsymbol{n}$ is the outward-pointing normal to the turnover curve, and

$$
\bar{h}=\frac{\pi S^{2}}{16 v_{n}^{2}} \quad \text { at } \quad t=\omega(x-X(t), y-Y(t))
$$

where $S$ is the coefficient of the square root in the leading-order outer velocity potential, as given in (5.1).

For axisymmetric impactors given by $z=f(r)-t$ where $r=\sqrt{(x-X(t))^{2}+(y-Y(t))^{2}}$, the system (6.1) can be solved using the method of characteristics subject to the boundary conditions

on

$$
\bar{u}=2 v_{n} \cos \theta, \quad \bar{v}=2 v_{n} \sin \theta, \quad \bar{h}=\frac{\pi \chi^{\prime}(d(T))^{2}}{8 d(T)}
$$

$$
x=X(T)+d(T) \cos \theta, \quad y=Y(T)+d(T) \sin \theta
$$

where $T>0$ parametrises time, $0 \leqslant \theta<2 \pi$ parametrises the angle around the turnover curve, $\chi(\sigma)$ is given by (4.11) and $v_{n}$ is given by (4.17).

If $\tau$ parametrises distance along a characteristic, then we find that $\bar{u}, \bar{v}$ and subsequently $\bar{h}$ are given by

$$
\bar{u}=2 v_{n} \cos \theta, \bar{v}=2 v_{n} \sin \theta, \bar{h}=\frac{\pi \chi^{\prime}(d(T))^{2}}{8 d(T)} \frac{J(0, \theta, T)}{J(\tau, \theta, T)},
$$

where

$$
t=\tau+T, \quad x=X(T)+\left(2 v_{n} \tau+d(T)\right) \cos \theta, \quad y=Y(T)+\left(2 v_{n} \tau+d(T)\right) \sin \theta
$$

and the Jacobian, $J(\tau, \theta, T)$, is given by

$$
\begin{aligned}
J(\tau, \theta, T)= & -4 v_{n} \frac{\partial v_{n}}{\partial T} \tau^{2}+2\left(2 v_{n}^{2}-\dot{d}(T) v_{n}-d(T) \frac{\partial v_{n}}{\partial T}\right. \\
& \left.-\dot{X}\left(v_{n} \cos \theta+\frac{\partial v_{n}}{\partial \theta} \sin \theta\right)+\dot{Y}\left(\cos \theta \frac{\partial v_{n}}{\partial \theta}-v_{n} \sin \theta\right)\right) \tau \\
& +2 v_{n} d(T)-\dot{X} d(T) \cos \theta-\dot{Y} d(T) \sin \theta-d(T) \dot{d}(T)
\end{aligned}
$$

We require the Jacobian to be bounded and nonzero. When the Jacobian vanishes, the solution becomes multivalued and the characteristics intersect. We will now illustrate this singularity formation by returning to the examples of $\S \S 4.3-4.4$.

\subsection{Splash sheet of an obliquely impacting cone}

For the constant speed oblique impact of a cone, the solution for the thickness of the splash sheet is given by

$$
\bar{h}=\frac{\beta T}{8} \frac{J(0, \theta, T)}{J(\tau, \theta, T)},
$$

where

$$
J(\tau, \theta, T)=\left(\frac{32}{\beta^{2} \pi^{2}}+\frac{16 U}{\beta \pi} \cos \theta+2 U^{2}\right) \tau+\frac{4 T}{\beta \pi}\left(\frac{4}{\beta \pi}+U \cos \theta\right) .
$$



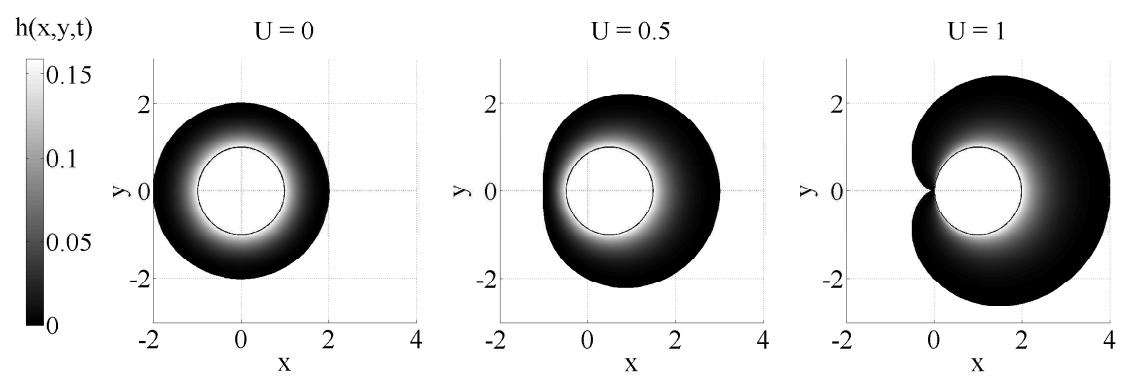

Figure 5. Cone splash sheet for (from left to right) $U=0,0.5,1$. The turnover curve is depicted by the bold circle in each plot. The figures represent a top-down view of the splash sheet between the touchdown curve and turnover curve. In each figure, the shading represents the thickness of the splash sheet. We can clearly see the breakdown of the splash sheet as we approach the critical value $U=1$. The touchdown curve forms a cusp, which touches the turnover curve at $\theta=\pi$, where $\theta=0$ is the direction of motion.

Clearly, $J(\tau, \theta, T)$ is bounded for all $\tau, T>0$ and $0 \leqslant \theta<2 \pi$. Now, as $\beta, \tau, T, U>0$, it is evident that

$$
\min _{\theta} J(\tau, \theta, T)=\left.J(\tau, \theta, T)\right|_{\theta=\pi}=2\left(\frac{4}{\beta \pi}-U\right)^{2} \tau+\frac{4 T}{\beta \pi}\left(\frac{4}{\beta \pi}-U\right) .
$$

Therefore, when $0<U<4 /(\beta \pi)$ we must have that $J(\tau, \theta, T)>0$ for all $\tau, T>0$, $0 \leqslant \theta<2 \pi$, so that the splash sheet solution is valid for all time. When $U=4 /(\beta \pi)$, $J(\tau, \theta, T) \equiv 0$ on the ray $\theta=\pi$ and the solution breaks down immediately when the turnover curve stops advancing, a consequence of the root of the splash sheet collapsing.

To visualise the breakdown, we consider the touchdown curve, that is the tip of the splash sheet, which is thrown out at time $t=0$. In the above solution, this corresponds to the curve of points where $\bar{h}=0$. We find that

$$
x=2 t \cos \theta\left(U \cos \theta+\frac{4}{\beta \pi}\right), y=2 t \sin \theta\left(U \cos \theta+\frac{4}{\beta \pi}\right),
$$

gives the equation of the touchdown curve parametrically. In particular, when $U=0$, this represents the circle

$$
x^{2}+y^{2}=4 d(t)^{2},
$$

which is consistent with the normal impact of a cone, and for $U=4 /(\beta \pi),(6.11)$ represents the cardioid

$$
x=\frac{4 t}{\beta \pi}(\cos 2 \theta+2 \cos \theta+1), y=\frac{4 t}{\beta \pi}(\sin 2 \theta+2 \sin \theta),
$$

with cusp at $\theta=\pi$. For all intermediate values of $U$, the touchdown curve is a limaçon.

To help visualise the breakdown, we plot the splash sheet for different values of $U$ in figure 5 . Since the cone admits a similarity solution in which distance scales linearly with time, $t$, it is sufficient to plot at one instant only. Moreover we have arbitrarily chosen $4 /(\beta \pi)=1$ to fix the critical velocity at $U=1$.

In figure 5 we see the nature of the breakdown. As we approach the critical value of $U$, a cusp forms on the touchdown curve, which touches the turnover curve on the ray $\theta=\pi$ when $U=1$. Thus, when the turnover curve stops advancing on this ray, the small aspect ratio assumption we made when deriving the splash sheet equations breaks down. We can check this: from (6.11), the extent of the splash sheet along the ray $\theta=\pi$ is 
given by

$$
|2 \cos \pi(U \cos \pi+1)-(U+\cos \pi)|=1-U .
$$

The maximum thickness of the splash sheet along that ray is on the turnover curve and is equal to $1 /(2 \pi)$. Clearly, as $U \uparrow 1$, the aspect ratio blows up.

We also note that at $U=1$, the turnover curve has stopped advancing for $\theta=\pi$, so that $(\bar{u}, \bar{v})=0$ at this point.

This breakdown is analogous to that found in the oblique small-deadrise impact of a two-dimensional wedge, in which we see the length of the trailing splash jet vanish as we approach the critical horizontal velocity, as described in Moore et al. (2012).

\subsection{Splash sheet of a blunt power-law body}

Recall that in the constant speed oblique impact of a blunt power-law body profile, the motion first breaks down at the critical time (4.24) on the ray $\theta=\pi$, the direction opposite the oblique motion. The outward normal speed of the turnover curve (4.23) can be written as

$$
v_{n}(\theta, t)=\frac{d(t)}{n t}+U \cos \theta
$$

so that requiring $v_{n}(\theta, t)>0$ on $\theta=\pi$, the condition that the turnover curve is advancing on this ray, gives

$$
\frac{d(t)}{n t}>U
$$

At breakdown, equality holds in the above statement.

Upon substituting into the general solution (6.7)-(6.8), we find that the splash sheet profile is defined by

$$
\begin{aligned}
& x=\left(2 v_{n} \tau+d(T)\right) \cos \theta+U T, \\
& y=\left(2 v_{n} \tau+d(T)\right) \sin \theta, \\
& \bar{h}=\frac{1}{\pi} n^{2} T^{2-1 / n}\left(\beta \mathrm{B}\left(\frac{n+2}{2}, \frac{n+2}{2}\right)\right)^{1 / n} \frac{J(0, \theta, T)}{J(\tau, \theta, T)},
\end{aligned}
$$

where

$$
\begin{aligned}
J(\tau, \theta, T)= & \frac{4(n-1)}{n^{2} T^{2}} d(T)\left(\frac{d(T)}{n T}+U \cos \theta\right) \tau^{2}+ \\
& 2\left(\frac{d(T)^{2}}{n T^{2}}+2 \frac{U d(T)}{n T} \cos \theta+U^{2}\right) \tau+d(T)\left(\frac{d(T)}{n T}+U \cos \theta\right) .
\end{aligned}
$$

This solution is valid for $0 \leqslant \tau, T<t_{c}, 0 \leqslant \theta<2 \pi$ provided that $J(\tau, \theta, T)$ is bounded and nonzero. We note that as $\bar{h}>0$ for all $\tau, \theta, T$ in these ranges and as $v_{n}$ is unbounded for $T=0$, the splash sheet extends to infinity. This is analogous to the infinite splash jet in two-dimensional blunt body impact.

Clearly the Jacobian is bounded for all $0 \leqslant \tau, T<t_{c}, 0 \leqslant \theta<2 \pi$. The equation (6.15) is a quadratic in $\tau$. As $n>1$, the coefficients of the quadratic and constant term are non-negative when $v_{n}>0$. Moreover, we can rewrite the coefficient of the $\tau$-term as

$$
2\left(\left(\frac{d(T)}{n T}+U \cos \theta\right)^{2}+\frac{(n-1) d(T)^{2}}{n^{2} T^{2}}\right),
$$

and hence, for $n>1$, this is also positive. Therefore, for $0 \leqslant \tau, T<t_{c}, 0 \leqslant \theta<2 \pi$, the Jacobian is also non-zero, so the solution is valid for all values in these ranges.

At breakdown on the turnover curve, $\tau=0$ and $\theta=\pi$ and thus the outward normal 
speed vanishes so that we deduce $J\left(0, \pi, t_{c}\right)=0$. Hence our solution is again no longer valid at breakdown.

We can interpret this physically by noting that the bicharacteristics of (6.1) are given by particle paths, viz.

$$
\frac{\partial x}{\partial \tau}=\bar{u}, \frac{\partial y}{\partial \tau}=\bar{v}, \frac{\partial t}{\partial \tau}=1 .
$$

At breakdown, we have $\bar{u}=\bar{v}=0$ on the turnover curve at $\theta=\pi$. Hence the bicharacteristics are parallel to the turnover curve at $\theta=\pi$. There is no longer any fluid entering the splash sheet at this point and our solution breaks down. So, we see a clear similarity to the breakdown of the splash jet problem in two-dimensional oblique blunt body impacts as described in Moore et al. (2012).

\section{Summary and discussion}

This work generalises oblique impact Wagner theory to three-dimensional body profiles. The leading-order outer problem is greatly simplified by working with the displacement potential, which reveals that the problem can be reduced to the corresponding normal impact model. Hence, given a solution for the leading-order turnover curve projection, leading-order outer free surface and leading-order displacement potential in the normal impact of the body $z=f(x, y)-Z(t)$, we are able to write down the corresponding leading-order turnover curve projection, leading-order outer free surface and leading-order displacement potential for the oblique entry of the body profile $z=f(x-X(t), y-Y(t))-Z(t)$ for oblique velocity components $X(t), Y(t)$ such that $\dot{X}, \dot{Y}=O(1)$. Due to the time dependence of the moving frame, more care has to be employed in deducing the leading-order outer velocity potential and pressure.

By solving the general leading-order axisymmetric normal impact problem, the solution can be written down for an arbitrary oblique impact. The turnover curve is simply a translation of the corresponding normal impact turnover curve. However, because the translation affects the crucial outward normal speed of the turnover curve, there is the possibility of the turnover curve ceasing to advance. If this occurs, an instability arises in a neighbourhood of the retreating turnover curve and our theory breaks down. Furthermore, we are no longer able to solve for the leading-order outer free surface if the turnover curve is retreating. These properties of breakdown are analogous to those seen due to a retreating turnover point in two-dimensional oblique impact problems. Moreover, at breakdown, the coefficients of the inverse square-root singularities in the leading-order outer velocity and pressure on the impactor vanish. This indicates a general breakdown of the asymptotic structure.

We have investigated breakdown for two specific examples of axisymmetric impacts with a constant oblique speed in the $x$-direction. For an obliquely impacting cone, which has a similarity solution, we find that provided the oblique speed is less than a critical value the solution remains valid for all time. However, at this critical value, the theory breaks down immediately. For a smooth axisymmetric power-law body, there exists a finite time at which the outward normal speed of the turnover curve vanishes for any nonzero oblique speed. In such cases, the leading-order outer pressure on the body becomes negative on part of the impactor prior to breakdown. This region grows in size as we approach breakdown, at which the isobar of zero pressure on the impactor touches the turnover curve. We explicitly showed that this was the case for the cone and the paraboloid. There is the possibility that cavitation occurs prior to breakdown, which is discussed further in the Appendix.

Assuming that cavitation can be ignored, we can solve the splash sheet problem. For 


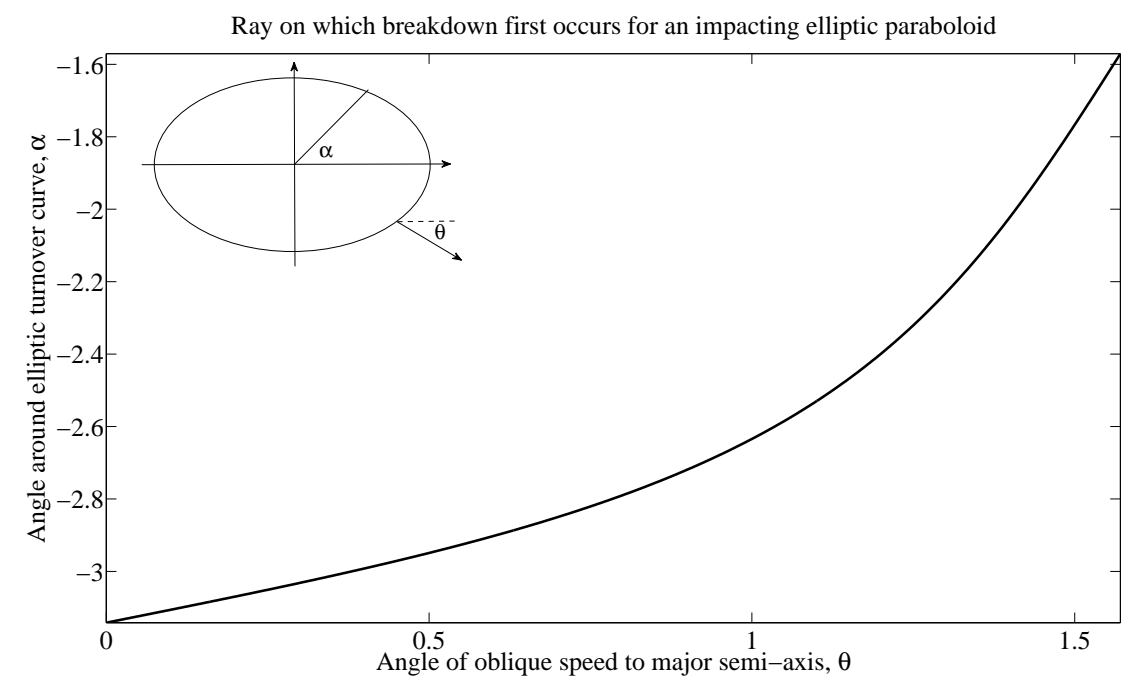

FiguRE 6. Illustrating breakdown for a non-axisymmetric body profile. The turnover curve for the impact of an elliptic paraboloid is an ellipse and the angle, $\alpha$, at which the breakdown of the leading-order Wagner theory first occurs on this ellipse is plotted as a function of the angle the oblique speed makes with the major semi-axis of the ellipse, $\theta$. Clearly, the breakdown does not always initiate in the direction opposite the motion. The eccentricity of the plotted ellipse is 0.933 .

the case of the cone with a constant oblique speed in the $x$-direction, the splash sheet has finite extent, terminating on the impactor at the touchdown curve. While the turnover curve is always a circle in the moving frame, for nonzero oblique impact speeds, the touchdown curve is a limaçon with minimum distance from the turnover curve on the ray in the direction opposite to the tangential motion. At the critical tangential speed, the touchdown curve becomes a cardioid with the cusp touching the turnover curve on the ray in the direction opposite to the tangential motion. The small aspect ratio assumption in the splash sheet becomes invalid on this ray.

In the case of a smooth power-law impactor, the splash sheet is thrown out to infinity at the moment of impact, so there is no touchdown curve. As we approach the critical time, the fluid entering the splash sheet at the turnover curve has vanishing speed. This form of breakdown in the splash sheet also holds for a more general (not necessarily axisymmetric) body profile.

Our results regarding the relationship between the leading-order outer oblique and normal impact problems hold for non-axisymmetric impactors. For example, for the constant speed oblique impact of an elliptic paraboloid, the normal impact of which is considered in Scolan \& Korobkin (2001), the turnover curve is an ellipse. Here, the normal impact problem is not axisymmetric and so we must consider the relationship between the horizontal velocity of the impactor and the orientation of the ellipse. In figure 6 we plot the angle at which breakdown first occurs on the turnover curve ellipse as a function of the angle between the oblique velocity and the major semi-axis of the ellipse. In particular, the breakdown only occurs in the direction directly opposite the motion when the oblique velocity is in the direction of one of the semi-axes of the ellipse. 


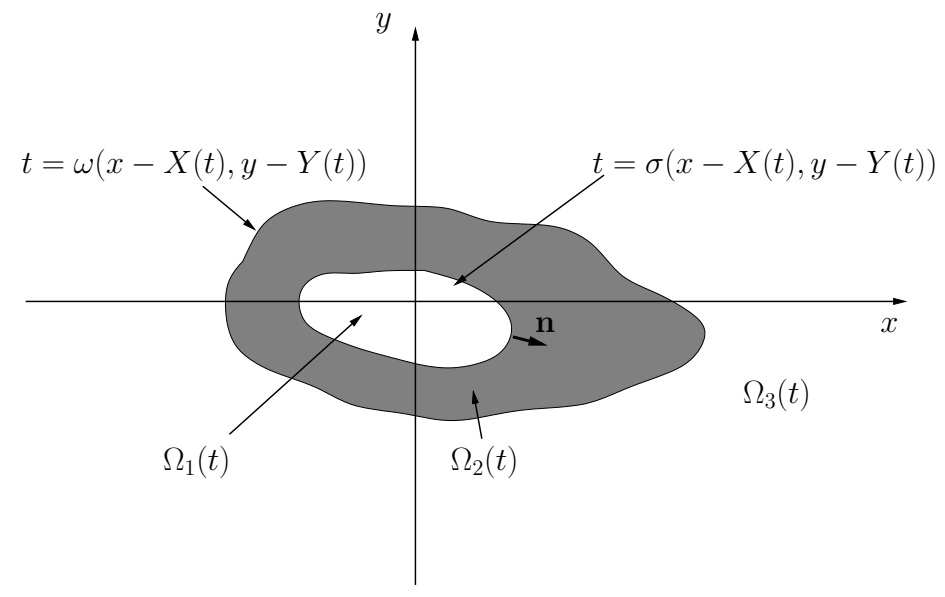

FIGURE 7 . The three regions of the leading-order outer problem with the introduction of a patch cavity on the impactor. $\Omega_{1}(t)$ represents the cavity, $\Omega_{2}(t)$ defines the remainder of the contact set and $\Omega_{3}(t)$ defines the non-contact set. The leading-order positions of the turnover curve and the edge of the patch cavity are defined by $t=\omega(x-X(t), y-Y(t))$ and $t=\sigma(x-X(t), y-Y(t))$ respectively. The outward-pointing normal to the cavity is denoted by $\boldsymbol{n}$.

\section{Appendix A. A model for cavitation}

We briefly outline the model that would need to be solved if cavitation is assumed to occur on the impactor. We suppose that the pressure required for cavitation to occur is given by $p_{c}<p_{\text {atm }}$ in dimensional coordinates, where $p_{\text {atm }}$ represents atmospheric pressure. We suppose that the pressure in any cavity takes this value. Then, provided

$$
\varepsilon \frac{p_{c}-p_{\text {atm }}}{\rho \dot{Z}(0)^{2}} \ll 1,
$$

the pressure in any cavity is given by $p=0$ in outer variables at leading order. Thus, when the leading-order outer pressure becomes negative in a subset of the contact region, we assume that a patch cavity forms on the impactor about the region of negative pressure. The dynamics and analysis of patch cavities is outlined in Howison et al. (1994). Our model is an adaptation of the discussion in Korobkin (2003) for a decelerating, normal, two-dimensional impact, to the oblique impact of the body profile $z=f(x-X(t), y-$ $Y(t))-Z(t)$.

At the first instance the pressure on the impactor becomes negative, we assume that a cavity grows from a single point. We assume the cavity thickness is much smaller than its dimensions in the $x$ - and $y$-directions so that in the leading-order outer problem, the cavity boundary conditions linearise onto the plane $z=0$. We sketch the key regions in the leading-order outer problem in figure 7. The leading-order turnover curve is again defined by $t=\omega(x-X(t), y-Y(t))$ and the leading-order boundary of the cavity is denoted by $t=\sigma(x-X(t), y-Y(t))$. We note that the patch cavity does not necessarily encompass the minimum of the impactor. The region $\Omega_{1}(t)$ defines the cavity on the impactor, that is $(x, y)$ such that $t>\sigma(x-X(t), y-Y(t))$. The region $\Omega_{2}(t)$ defines the rest of the contact set, that is $(x, y)$ such that $\omega(x-X(t), y-Y(t))<t<\sigma(x-$ $X(t), y-Y(t))$. Finally, $\Omega_{3}(t)$ defines the non-contact set, that is $(x, y)$ such that $t<$ $\omega(x-X(t), y-Y(t))$.

The leading-order outer velocity potential in the fluid, $\phi(x, y, z, t)$, the leading-order outer free surface, $h(x, y, t)$, and the leading-order thickness of the cavity, $H(x, y, t)$, must 
then satisfy:

$$
\begin{aligned}
\nabla^{2} \phi & =0 & & \text { in } \quad z<0, \\
\phi & =0 & & \text { on } \quad z=0,(x, y) \in \Omega_{3}(t), \\
\frac{\partial \phi}{\partial z} & =\frac{\partial h}{\partial t} & & \text { on } \quad z=0,(x, y) \in \Omega_{3}(t), \\
\frac{\partial \phi}{\partial z} & =-\dot{Z}-\dot{X} f_{1}^{\prime}-\dot{Y} f_{2}^{\prime} & & \text { on } \quad z=0,(x, y) \in \Omega_{2}(t), \\
\frac{\partial \phi}{\partial z} & =-\dot{Z}-\dot{X} f_{1}^{\prime}-\dot{Y} f_{2}^{\prime}-\frac{\partial H}{\partial t} & & \text { on } \quad z=0,(x, y) \in \Omega_{1}(t),
\end{aligned}
$$

with the far-field conditions

$$
\begin{aligned}
& \phi \rightarrow O(1 / R) \quad \text { as } \quad R=\left(x^{2}+y^{2}+z^{2}\right)^{1 / 2} \rightarrow \infty \\
& h \rightarrow 0 \quad \text { as } \quad\left(x^{2}+y^{2}\right)^{1 / 2} \rightarrow \infty
\end{aligned}
$$

We still require $\phi$ to have a square-root singularity in distance from the turnover curve as we approach it in any perpendicular plane. Finally, the Wagner condition still holds at the turnover curve, as given in (3.6).

In addition to this, we require two further pieces of information to determine the location and size of the cavity. As described above, we must also have $p(x, y, 0, t)=0$ for $(x, y) \in \Omega_{1}(t)$ to leading-order. We take the second condition as in Korobkin (2003), namely that the pressure close to the edge of the cavity is continuously differentiable (that is we seek the solution with minimal singularity). Hence, we require that $\partial p / \partial n \rightarrow 0$ as we approach the cavity boundary, $t=\sigma(x-X(t), y-Y(t))$. Note that this forces $\partial^{2} H / \partial t^{2}$ to be bounded at the cavity edge.

It is evident that the introduction of a patch cavity makes analysis of the leading-order outer problem much more complicated. Moreover, changing to the displacement potential form of the problem does not appear to give any direct benefits. 


\section{REFERENCES}

Armand, J.-L. \& Cointe, R. 1987 Hydrodynamic impact analysis of a cylinder. ASME J. Offshore Mech. Arc. Eng. 111, 109-114.

Chekin, B. S. 1989 The entry of a wedge into an incompressible fluid. Journal of Applied Mathematics and Mechanics 53 (3), 300-307.

Dobrovol'skaya, Z. N. 1969 On some problems of similarity flow of fluid with a free surface. J. Fluid Mech. 36 (4), 805-829.

Garabedian, P. R. 1953 Oblique water entry of a wedge. Communications on Pure and Applied Mathematics 6 (2), 157-165.

Howison, S. D., Morgan, J. D. \& Ockendon, J. R. 1994 Patch cavitation in flow past a rigid body. Bubble Dynamics and Interface Phenomena 3, 219-226.

Howison, S. D., Ockendon, J. R. \& Oliver, J. M. 2004 Oblique slamming, planing and skimming. J. Engg. Math. 48, 321-337.

Howison, S. D., OCKendon, J. R. \& WiLson, S. K. 1991 Incompressible water-entry problems at small deadrise angles. J. Fluid Mech. 222, 215-230.

Judge, C., Troesch, A. \& Perlin, M. 2004 Initial water impact of a wedge at vertical and oblique angles. J. Engg Math. 48 (3), 279-303.

Korobkin, A. A. 1982 Formulation of penetration problem as a variational inequality. Din. Sploshnoi Sredy 58, 73-79.

Korobkin, A. A. 1988 Inclined entry of a blunt profile into an ideal fluid. Fluid Dynamics $23(3), 443-447$.

Korobkin, A. A. 2003 Cavitation in liquid impact problems. Fifth Intl. Symp. on Cavitation, Osaka, 1-4 November 2003 .

Miloh, T. 1991 On the oblique water-entry problem of a rigid sphere. J. Engg. Math. 25 (1), $77-92$.

Moore, M. R., Howison, S. D., Ockendon, J. R. \& Oliver, J. M. 2012 A note on oblique water-entry. Under consideration for J. Engg. Math. .

Oliver, J. M. 2002 Water entry and related problems. DPhil Thesis, University of Oxford.

Oliver, J. M. 2007 Second-order wagner theory for two-dimensional water-entry problems at small deadrise angles. J. Fluid Mech. 572, 59-85.

Scolan, Y.-M. \& Korobkin, A. A. 2001 Three-dimensional theory of water ipact. part 1. inverse wagner problem. J. Fluid Mech. 440, 293-326.

Semenov, Y. A. \& Yoon, B.-S. 2009 Onset of flow separation for the oblique water impact of a wedge. Phys. of Fluids 21, 1121031-11.

Shiffman, M. \& Spencer, D.C. 1951 The force of impact on a cone striking a water surface (vertical entry). Communications on Pure and Applied Mathematics 4 (4), 379-417.

Sneddon, I. N. 1966 Mixed Boundary Value Problems in Potential Theory. North-Holland.

VON KÁRMÁn, T. 1929 The impact of seaplane floats during landing. NACA TN 321.

WAGner, H. 1932 Über Stoß- und Gleitvorgänge an der Oberfläche von Flüssigkeiten. Z. angew. Math. Mech. 12, 193-215. 



\section{RECENT REPORTS}

12/08 Qualitative Analysis of an Integro-Differential Equation Model of Periodic Chemotherapy

Jaina

Byrne

12/09 Modeling Stem/Progenitor Cell-Induced Neovascularization and

Jain Oxygenation

Moldovan

Byrne

12/10 Allee Effects May Slow the Spread of Parasites in a Coastal Marine Ecosystem

Krkošek

Connors

Lewis

Poulin

12/11 Parasite spill-back from domestic hosts may induce an Allee effect in wildlife hosts

Krkošek

Ashander

Lewis

12/12 Modelling temperature-dependent larval development and sub-

Wheeler sequent demographic Allee effects in adult populations of the alpine butterfly Parnassius smintheus

Bampfylde

Lewis

12/13 Putting "space" back into spatial ecology

Fortin

Peres-Neto

Lewis

12/14 Wildlife disease elimination and density dependence

Potapova

Merrill

Lewis

12/15 Spreading Speed, Traveling Waves, and Minimal Domain Size in Impulsive Reaction-diffusion Models

Lewis

$\mathrm{Li}$

12/16 MCMC methods for functions modifying old algorithms to make

Cotter them faster

Roberts

Stuart

White

12/17 Weyl Geometry and the Nonlinear Mechanics of Distributed Point

Yavari Defects

Goriely

12/18 A note on oblique water entry

Moore

Howison

Ockendon

Oliver

12/19 Calculus on surfaces with general closest point functions

März

Macdonald

12/20 Multiple equilibria in a simple elastocapillary system

Taroni

Vella

Hubbard

Byrne 
12/23 Moment-based formulation of NavierMaxwell slip boundary conditions for lattice Boltzmann simulations of rarefied flows in microchannels

Dellar

12/24 Correspondence between one- and two-equation models for solute transport in two-region heterogeneous porous media

Davit

Wood

Debenest

Quintard

12/25 Rolie-Poly fluid flowing through constrictions: Two distinct instabilities

Reis

Wilson

12/26 Age related changes in speed and mechanism of adult skeletal

Collins-Hooper muscle stem cell migration

Woolley

Dyson

Patel

Potter

Baker

Gaffney

Maini

Dash

Patel

12/27 The interplay between tissue growth and scaffold degradation in

ODea engineered tissue constructs

Osborne

El Haj

Byrne

Waters

12/28 Non-linear effects on Turing patterns: time oscillations and

Aragon chaos.

Barrio

Woolley

Baker

Maini

12/29 Colorectal Cancer Through Simulation and Experiment

Kershaw

Byrne

Gavaghan

Osborne

12/30 A theoretical investigation of the effect of proliferation and adhe-

Mirams sion on monoclonal conversion in the colonic crypt

Fletcher

Maini

Byrne

12/31 Convergent evolution of spiny mollusk shells points to elastic en-

Chirat ergy minimum

Moulton

Shipman

Goriely 
Copies of these, and any other OCCAM reports can be obtained from:

Oxford Centre for Collaborative Applied Mathematics Mathematical Institute

24 - 29 St Giles'

Oxford

OX1 3LB

England

www.maths.ox.ac.uk/occam 